\title{
Erratum to: Paenibacillus beijingensis sp. nov., a nitrogen- fixing species isolated from wheat rhizosphere soil
}

\author{
Li-Ying Wang $\cdot$ Ji Li • Qing X. Li • \\ San-Feng Chen
}

Published online: 11 January 2014

(C) Springer Science+Business Media Dordrecht 2014

\section{Erratum to: Antonie van Leeuwenhoek (2013) 104: 675-683 DOI 10.1007/s10482-013-9974-5}

Subsequent to the publication of the above paper it has been brought to our attention that the name proposed in the paper for the taxon represented by strain $\mathrm{BJ}-18^{\mathrm{T}}$ is not appropriate as the same name for a taxon with a different type strain has been effectively published previously (Gao M. et al. 2012. Antonie van Leeuwenhoek 102: 689-694. doi: 10.1007/s10482-0129767-2). We apologise for this confusion and here propose a new name for the taxon represented by strain BJ- $18^{\mathrm{T}}$ :

The online version of the original article can be found under doi:10.1007/s10482-013-9974-5.

L.-Y. Wang · S.-F. Chen $(\bowtie)$

State Key Laboratory for Agrobiotechnology, China

Agricultural University, Beijing 100193, China

e-mail: chensf@cau.edu.cn

L.-Y. Wang

e-mail: wangliying_313@163.com

J. Li

College of Resources and Environmental Engineering,

China Agricultural University, Beijing 100193, China

Q. X. Li

Department of Molecular Biosciences and Bioengineering, University of Hawaii, Honolulu, HI 96822, USA

\section{Description of Paenibacillus triticisoli sp. nov.}

Paenibacillus triticisoli (tri.ti.ci.so'li. N.L. n. triticum wheat; L. n. solum soil; N.L. gen. n. triticisoli of the soil surrounding a wheat plant, the source of the type strain).

The description is as given for "Paenibacillus beijingensis strain $\mathrm{BJ}-18^{\mathrm{T}}$ ” in Wang et al. (2013) Antonie van Leeuwenhoek 104: 675-683.

The type strain BJ-18 ${ }^{\mathrm{T}}\left(=\mathrm{DSM} 25425^{\mathrm{T}}=\mathrm{CGMCC}\right.$ $\left.1.12045^{\mathrm{T}}\right)$.

Acknowledgments We thank Professor George Garrity for his advice on resolving the nomenclature of this taxon. 